\title{
OP90
}

\section{TEST METHOD OF LUMINANCE DYNAMIC RANGE FOR HDR CAMERA WITH CMOS IMAGE SENSOR \\ ChangHui Ye et al.}

DOI 10.25039/x46.2019.OP90

from

CIE x046:2019

Proceedings

of the

29th CIE SESSION

Washington D.C., USA, June 14 - 22, 2019

(DOI 10.25039/x46.2019)

The paper has been presented at the 29th CIE Session, Washington D.C., USA, June 14-22, 2019. It has not been peer-reviewed by CIE.

(C) CIE 2019

All rights reserved. Unless otherwise specified, no part of this publication may be reproduced or utilized in any form or by any means, electronic or mechanical, including photocopying and microfilm, without permission in writing from CIE Central Bureau at the address below. Any mention of organizations or products does not imply endorsement by the CIE.

This paper is made available open access for individual use. However, in all other cases all rights are reserved unless explicit permission is sought from and given by the CIE.

CIE Central Bureau

Babenbergerstrasse 9

A-1010 Vienna

Austria

Tel.: +4317143187

e-mail: ciecb@cie.co.at

www.cie.co.at 


\title{
TEST METHOD OF LUMINANCE DYNAMIC RANGE FOR HDR CAMERA WITH CMOS IMAGE SENSOR
}

\author{
Chang Hui Ye ${ }^{1}$, Jeong Ah Hwang ${ }^{1}$, Dong-Hoon Lee ${ }^{2}$, Seongchong Park ${ }^{2}$ \\ ${ }^{1}$ Pixelplus Co.LTD., Suwon, KOREA, ${ }^{2}$ Korea Research Institute of Standards and Science (KRISS), \\ Daejeon, SOUTH KOREA \\ ych@pixelplus.com
}

DOI 10.25039/x46.2019.OP90

\begin{abstract}
We present a test method of luminance dynamic range for a CMOS-based HDR camera, which provides a practical solution with a cost-effective instrumentation. The method is based on the separate measurements of the relative linearity and the absolute scaling. The relative linearity measurement is performed in the illuminance mode for the CMOS image sensor without lens system by using a collimated beam. The absolute scaling measurement is performed for the camera including the lens system against an integrating sphere source at a moderate luminance level. Combining these two measurements, we obtain a linearity plot of the camera reading signal against object luminance, from which the saturation luminance and the noise equivalent luminance are determined. The method is demonstrated for a HDR camera with a dynamic range of 20,000:1.
\end{abstract}

Keywords: image sensor, luminance dynamic range, HDR camera.

\section{Introduction}

Now a days, most high dynamic range (HDR) cameras are realized based on the CMOS image sensor architecture, because CMOS image sensors provide enough photo-electron capacity to realize the world luminance into single digitized image. The photo-electron capacity, which is related with the dynamic range of CMOS image sensors, has been improved by various techniques, e.g., by using lateral overflow capacitors inside the pixel structure, or by using the intermediate voltage for reading electrons of photodiodes with multiple exposure times. The key performance of such HDR image sensors is the dynamic range of operation. To determine the numerical value of the dynamic range, many parameters affecting the dynamic range such as ADC bit depth, ratio of conversion gain, and multiple exposures should be carefully considered or controlled.

To test the optical dynamic range of a HDR camera in the unit of luminance, one needs a uniform source, typically an integrating sphere-based broadband source, whose luminance can be varied in a wide range from $10^{-3} \mathrm{~cd} / \mathrm{m}^{2}$ to $10^{5} \mathrm{~cd} / \mathrm{m}^{2}$ without changing its spectrum. Such a HDR luminance standard source might be available in a national metrology institute or in a testing laboratory. However, the industry requires a more practical test method without a sophisticated instrumentation. In this work, we propose an alternative test method for luminance dynamic range of a HDR camera, which can be realized in a simple and compact experimental setup.

\section{Experimental Methods}

The key concept of the proposed method is to separate the relative linearity measurement and the scaling measurement of the luminance response. The linearity measurement is performed against a collimated beam for a camera without the imaging optics (illuminance mode), while the scaling measurement is performed against an integrating sphere source for a camera with the imaging optics attached (luminance mode). The link of the two measurements is realized at a fixed readout value of the CMOS sensor. The advantage of this method is that the saturation level of an HDR image sensor can be easily reached in the illuminance mode at only a moderate radiant power with a collimated beam. In this section, the experimental setup and methods for the proposed dynamic range test are described. 


\subsection{Measurement Setup}

For the relative linearity measurement, we used the measurement setup for spectral responsivity of image sensors for a collimated beam incidence, as shown in Figure 1 (YE, 2018). Instead of operating the tunable monochromatic source, the monochromator is set to zero wavelength so that the $250-\mathrm{W}$ quartz-tungsten-halogen lamp forms a collimated white beam incident on the image sensor under test at 0 degree. A variable neutral-densitiy (ND) filter is used to control the radiant power level of the white beam, which is monitored by the reference photodiode (REF) through a beam splitter. In this case, the REF acts as a linearity reference. The linearity of the REF is separately verified from $1 \mathrm{pA}$ to $1 \mathrm{~mA}$ range based on the flux addition method (SHIN, 2014). As a result of the linearity measurement, we obtain a plot of the digital number (DN) readings of the test CMOS sensor, averaged for a specified area of interest, as a function of the REF photocurrent reading in $A$.

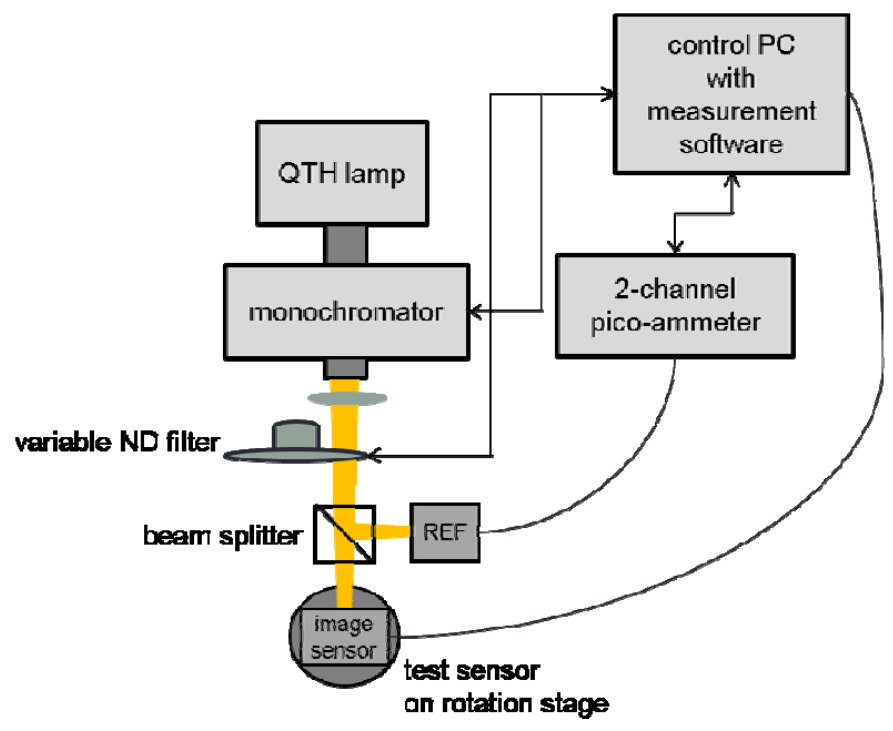

Figure 1 - Experimental setup for measurement of spectral responsivity of image sensors for a collimated beam incidence, which is used for the relative linearity measurement in this work.

For the scaling measurement, a commercially available integrating sphere source is used, which provides a continuously variable luminance at a spectrum close to the CIE illuminance A. The test CMOS camera with the imaging optics attached is operated in the same conditions as for the linearity measurement. At a specific value of the DN reading, the luminance value of the integrating sphere source is determined, which determines the luminance response of the camera. Both the linearity measurement and the scaling measurement are performed for each channel of the CMOS camera separately.

\subsection{Test Sample}

For testing the optical dynamic range, we tested the dual-conversion-gain type model of HDR colour image sensor manufactured by Pixelplus. Dual-conversion-gain type can be operated in two different modes, HS (High Sensitivity) and LS (Low Sensitivity), which have different responsivities. A high dynamic range is achieved by combining the different sensitivity modes into one response signal considering the ratio between the gains of HS mode and LS mode to extend the saturation level, as depicted in Figure 2. This technology is advantageous for getting a clearer motion picture compared with the multiple-exposure type, as two images with different sensitivities are taken in the same exposure time.

The sensor under test has an array of $1280 \times 960$ pixels with a pixel size of $3.75 \mu \mathrm{m} \times$ $3.75 \mu \mathrm{m}$ (model PK2130). Figure 3 shows the quantum efficiency of the test image sensor as a function of wavelength, which is determined from the measured spectral responsivity (Ye, 2017). Four different channels are available, which are denoted as R, Gr, Gb, and B. The dynamic range test is performed for DN readings of each channel separately. For the dynamic range test, the saturation luminance and the noise equivalent luminance are specified for the LS and HS mode, respectively, to specify the maximum achievable range. 
(a)

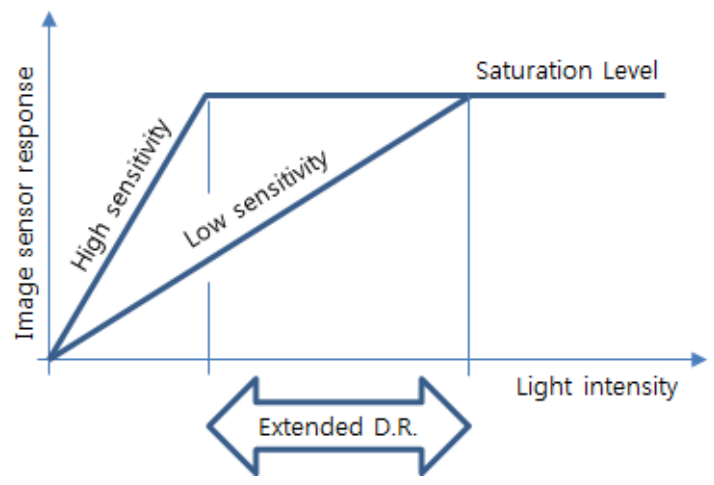

(b)

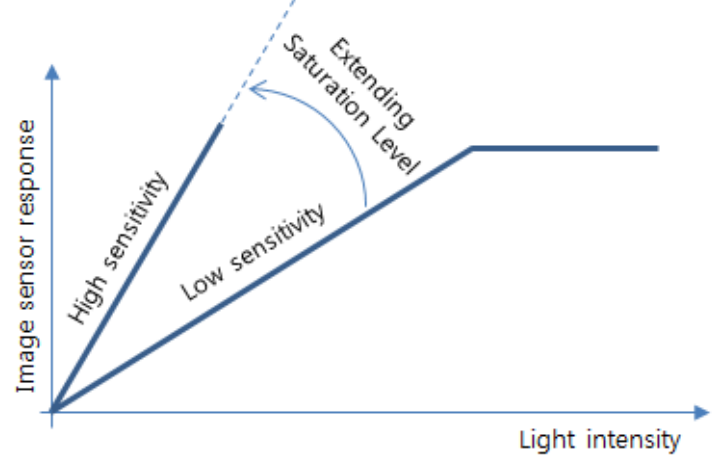

Figure 2 - Illustration of HDR image sensor response for (a) each response of dual sensitivity mode and (b) overcoming device saturation level by using lower sensitivity response combining into high sensitivity response.

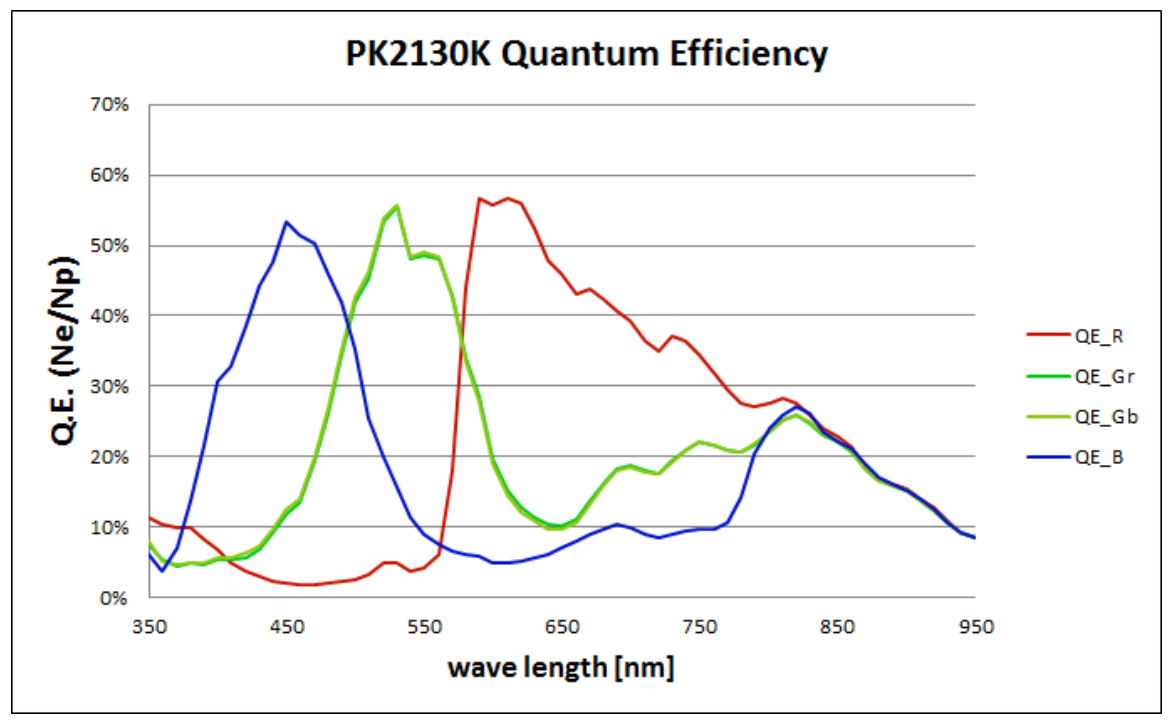

Figure 3-Quantum efficiency of the test image sensor.

\subsection{Test Method}

From the linearity measurement, we obtain a plot of the DN reading of the test sensor as a function of the photocurrent reading of the REF photodiode. By applying the luminance response in the unit of $\mathrm{DN} /\left(\mathrm{cd} / \mathrm{m}^{2}\right)$ determined from the scaling measurement, we can convert the linearity plot of DN against photocurrent to a plot DN against incident luminance. From the linearity plot as a function of luminance, we can determine the saturation luminance, at which the luminance response becomes zero.

The relative linearity measurement is performed for the CMOS image sensor without imaging optics against a collimated beam in the illuminance mode, while the scaling measurement is performed for the camera including imaging optics in the luminance mode. The linking of these two different modes is based on the camera equation describing image plane irradiance $E_{v}$ for extended Lambertian source (GRANT, 2011):

$$
E_{v}=\frac{\tau_{0} \tau_{a t m} \pi f_{v} L_{v} \cos ^{4} \theta}{4(f / \#)^{2}(1+m)^{2}}
$$

Here, $L_{v}$ is the source luminance; $\tau_{0}$ and $\tau_{a t m}$ are the transmittance of the optical system and of the atmosphere, respectively; $f_{v}$ is a vignetting factor, $\theta$ is the incident angle, $m$ is the 
magnification, and $f / \#$ is the f-number of the imaging optics. As long as the specifications of the imaging optics are fixed, the proportionality between the illuminance on the image sensor and the luminance of the Lambertian source is confirmed in Eq. (1) at a fixed incident angle. Therefore, the luminance response measured against an integrating sphere can be used to scale the linearity plot measured in the illuminance mode.

Next to the saturation luminance, we need to know also the noise equivalent luminance (NEL), at which the DN reading signal is the same as the noise count. The test sensor has a very high sensitivity so that the low signal level could not be precisely controlled by using the variable ND filter upon the white light beam. Therefore, we used the monochromatic light at the wavelengths where each color channel has its maximum quantum efficiency (see Figure 3 ). In this way, the linearity measurement in the reading range of a few DN could be also realized. The noise count of the CMOS image sensor at each signal reading is determined from the statistics among the pixels based on the following equation (JANESICK, 2007):

$$
\sigma_{\text {total }}(D N)=\left\{\frac{\sum_{i=1}^{N_{P I X}}\left[S_{i}(D N)-S(D N)\right]^{2}}{N_{P I X}}\right\}^{1 / 2}
$$

Here, $S_{i}(D N)$ is the reading signal of the $i$-th pixel without offset, and $S(D N)$ is the averaged value among the total number of pixel $N_{P I X}$ without offset. In this noise, all the components of read noise, shot noise, and fixed pattern noise are included. Note that the read noise originates from the purely electrical property of the sensor and independent upon the incident luminance. NEL is the luminance at which the signal reading is the same as the noise count.

Finally, the dynamic range is determined as the ratio of the saturation luminance over the NEL, often expressed in the log scale with a base of 20.

\section{Results and Discussion}

Figure 3 shows the results of the relative linearity measurement for the test image sensor in the LS mode by using a collimated white beam (AVGSIG: average signal). The DN reading signal averaged over $100 \times 100$ pixels of the specified area of interest is plotted as a function of the photocurrent of the REF photodiode. Note that the REF photocurrent is proportional to the illuminance on the sensor. The REF photocurrent is the converted to the luminance scale based on the scaling measurement at the integrating sphere source at a luminance level of approximately $500 \mathrm{~cd} / \mathrm{m}^{2}$. The resulting linearity plot against the luminance is shown in Figure 4. From this result, the saturation luminance is determined to be $4200 \mathrm{~cd} / \mathrm{m}^{2}, 6000 \mathrm{~cd} / \mathrm{m}^{2}$, and $8000 \mathrm{~cd} / \mathrm{m}^{2}$ for the R, G, and B channels, respectively.

The noise equivalent luminance is measured in the HS mode. Figure 5 shows the results in the HS mode. In this measurement, the average reading signals are recorded by subtracting the offset values (AVSWOO: average signal without offset). To determine the noise equivalent luminance, the total noise count is plotted together with the reading signal, as shown in Figure 6 for the green $(\mathrm{Gr})$ channel. The trace TOTALN corresponds to the total noise count calculated with Eq. (2). The signal and noise traces coincide at a luminance of $0.3 \mathrm{~cd} / \mathrm{m}^{2}$.

Consequently, for the Gr channel, we obtain a luminance dynamic range of $20,000: 1$, or $86 \mathrm{~dB}$ in the log scale with a base of 20. All the measurements are performed at an integration time as $1 / 1000 \mathrm{sec}$. This number should be compared with the range of the output digital number after combining from LS and HS signals to the 14-bit signal. The maximum output digital number for 14 bits is $16,384 \mathrm{DN}$ and the minimum read out noise of the ADC is $0.2887 \mathrm{DN}$ according to other references (JANESICK, 2007). The test result of the dynamic range is well within the maximum range given by $16,384: 0.2887=56,751: 1$. 


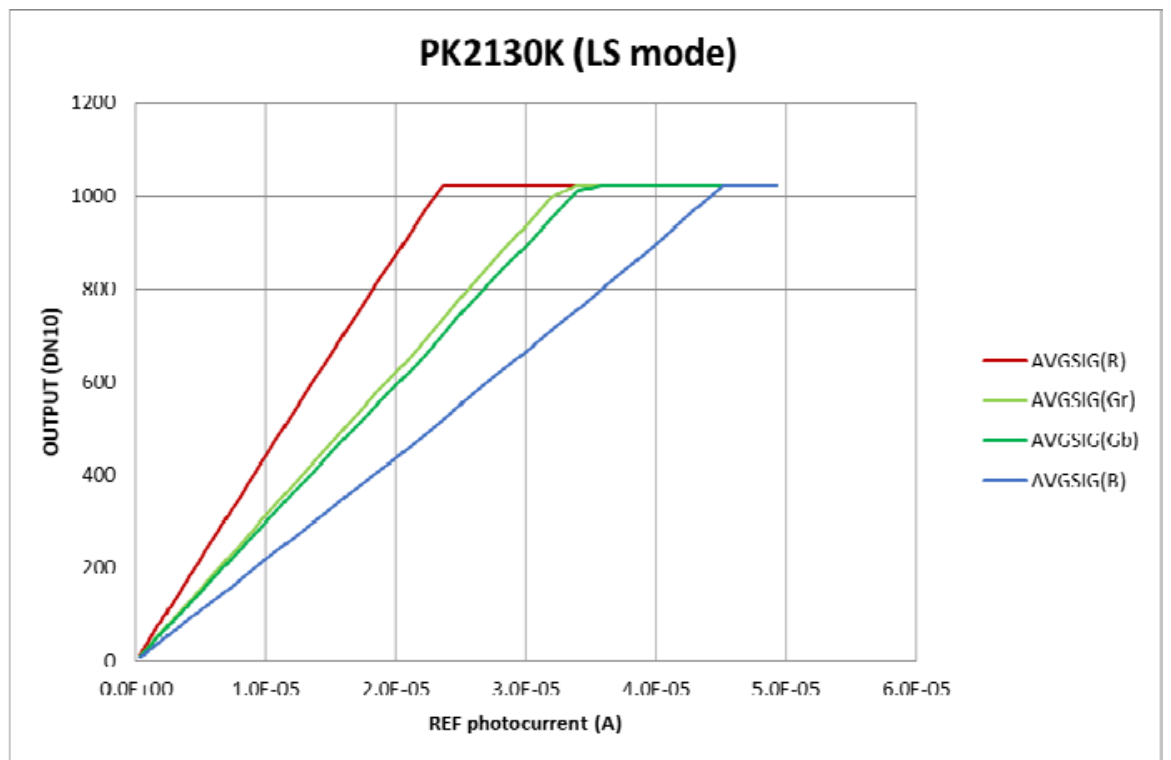

Figure 3 - Measurement result of the image sensor output in the LS mode as a function of luminance the photocurrent of the reference photodiode proportional to the illuminance on the sensor.

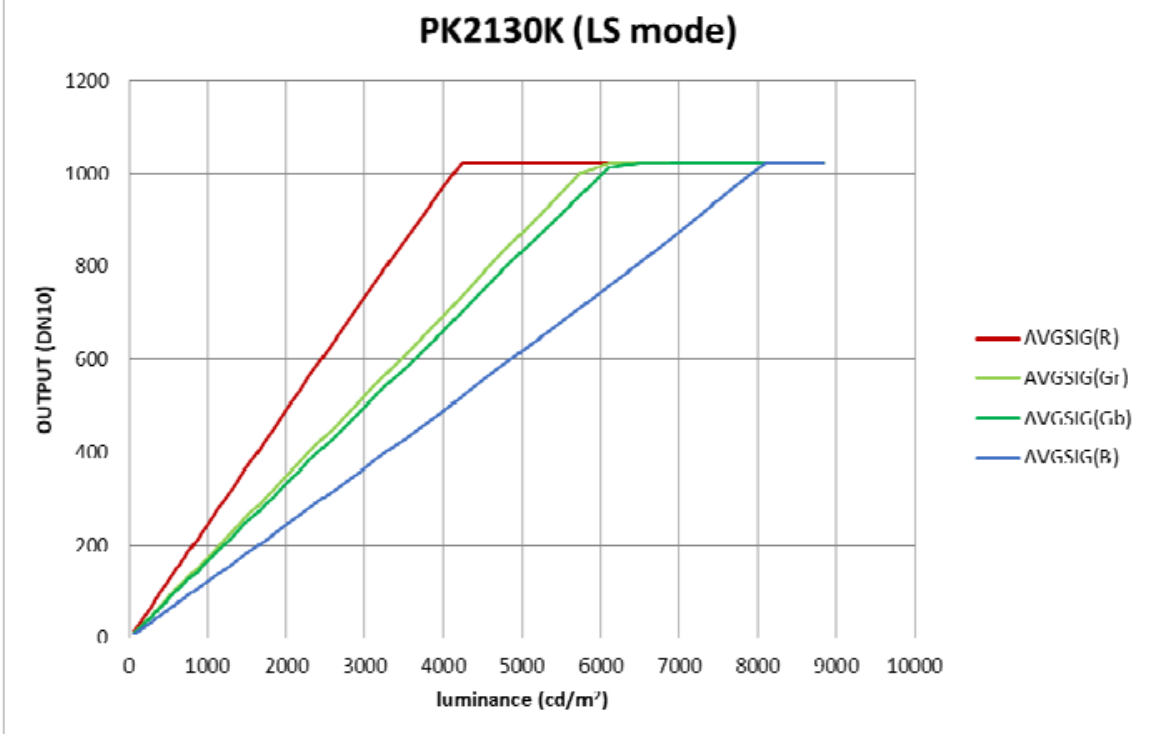

Figure 4 - Linearity plot of the test camera in the LS mode against luminance, which is converted from Figure 3 based on the scaling measurement. 


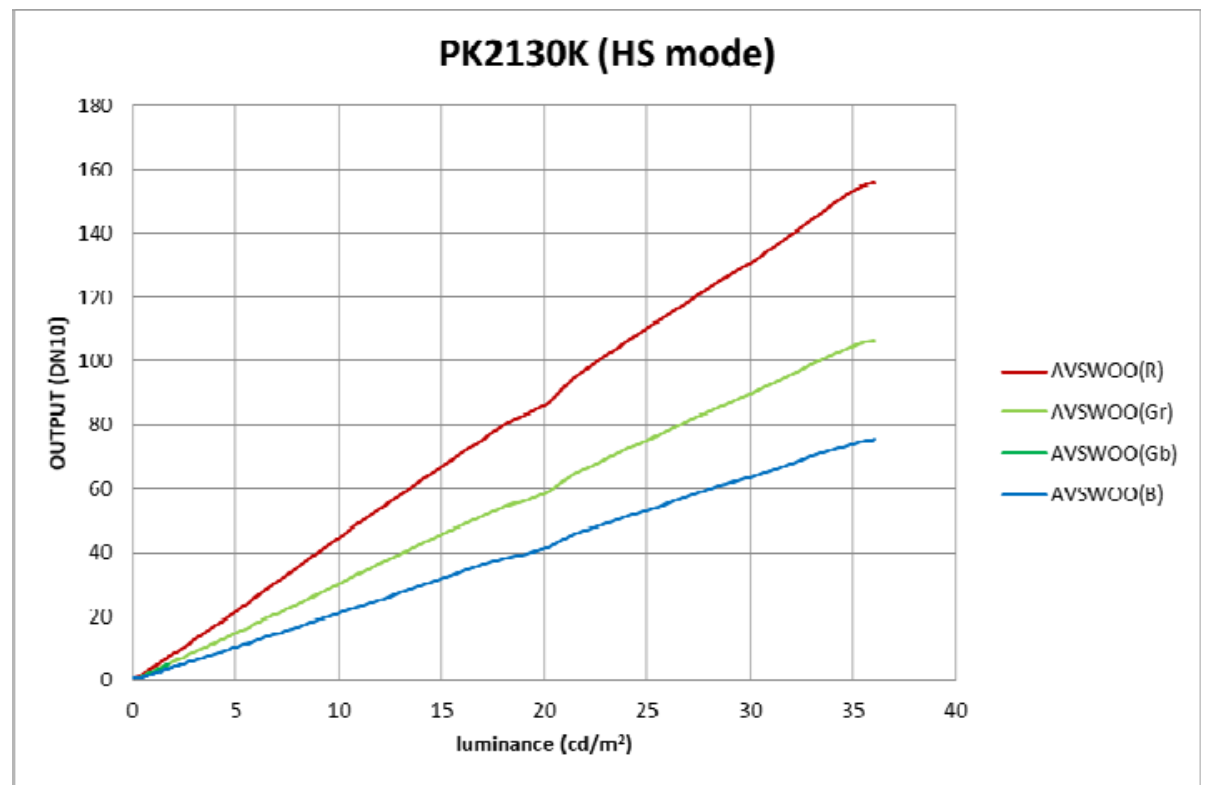

Figure 5 - Linearity plot of the test camera in the HS mode against luminance.

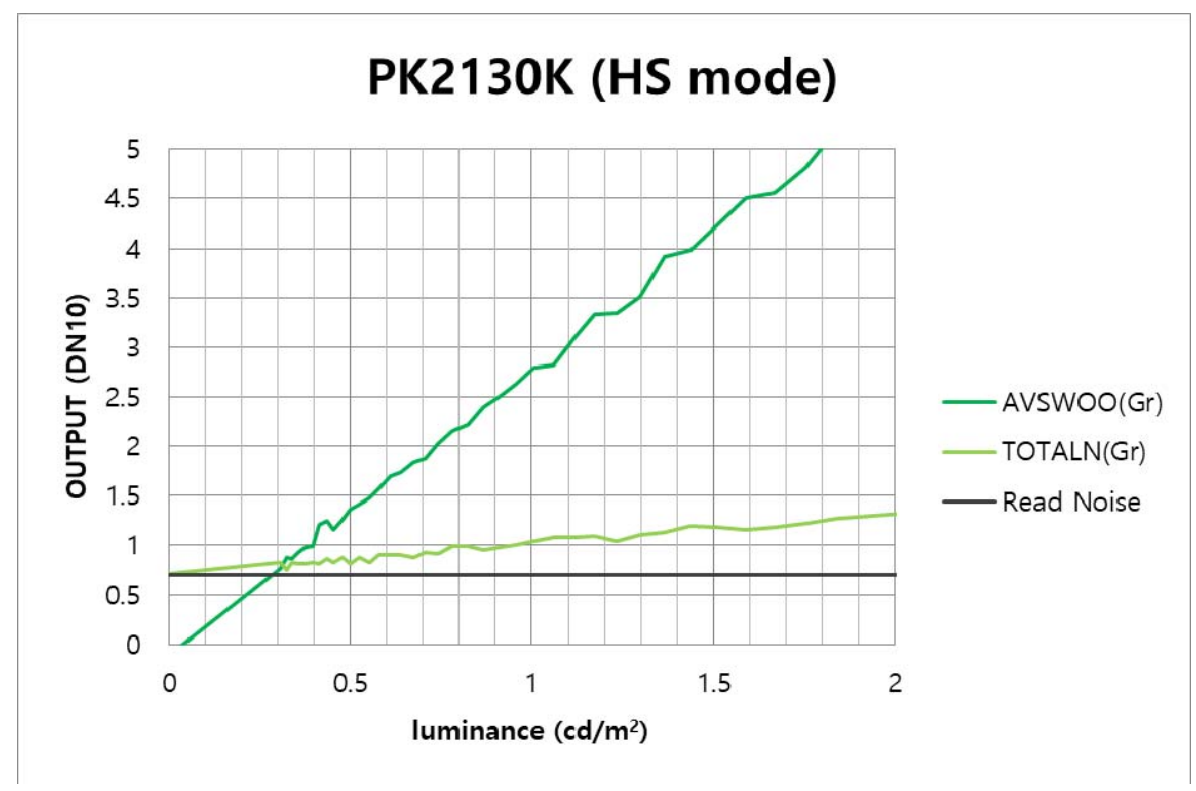

Figure 6 - Linearity plot of the test camera in the HS mode against luminance in the very low signal level for the determination of the noise equivalent luminance.

\section{Conclusion}

In this work, we demonstrated a practical test method of luminance dynamic range for a HDR camera based on CMOS image sensor. The method is based on the fixed relation between the illuminance on the image sensor and luminance of the source converted by an imaging optics.

The relative linearity measurement is performed for the image sensor without lens system by using a collimated white beam combined with a continuously variable ND filter. As the reference for the linearity measurement, a photodiode with a beam splitter is used. In this way, the saturation of the image sensor signal could be achieved at a relatively low power level. For the low signal level, the monochromatic beam at the wavelengths of the maximum quantum efficiency for each channel is used to more precisely control the light power. The 
linearity plot is then scaled against luminance by an additional measurement against an integrating sphere source at a low luminance level.

The method is applied to a HDR camera model of PIXELPLUS. At the green channel, a dynamic range of $20,000: 1$ or $86 \mathrm{~dB}$ could be successfully tested. The method is of practical importance as it requires no sophisticated instrumentation such as a luminance source variable in a wide range from $10^{-3} \mathrm{~cd} / \mathrm{m}^{2}$ up to $10^{5} \mathrm{~cd} / \mathrm{m}^{2}$. The evaluation or verification of the accuracy of the method is in progress.

\section{References}

GRANT, B. G. 2011. Field guide to radiometry. SPIE Field Guides Volume FG23. Washington: SPIE.

JANESICK, J. R. 2007. Photon transfer $D N \rightarrow \lambda$. Washington: SPIE.

SHIN, D.-J. et al. 2014. High-accuracy measurement of linearity of optical detectors based on flux addition of LEDs in an integrating sphere, Metrologia, 51, 25-32.

YE, C. H. et al. 2017. Influence of crosstalk on spectral responsivity of colour image sensors, Proc. CIE x044:2017.

YE, C. H. et al. 2018. Optical system design for characterization of photo-response nonuniformity (PRNU) of CMOS image sensors, Proc. SPIE 10746, Novel Optical Systems Design and Optimization XXI, 107460I. 